\title{
Prealbumin/CRP Based Prognostic Score, a New Tool for Predicting Metastasis in Patients with Inoperable Gastric Cancer
}

\author{
Ali Esfahani, ${ }^{1}$ Nima Makhdami, ${ }^{2}$ Elnaz Faramarzi, ${ }^{3}$ Mohammad Asghari Jafarabadi, \\ Alireza Ostadrahimi, ${ }^{5}$ Mousa Ghayour Nahand, ${ }^{6}$ and Zohreh Ghoreishi ${ }^{5}$ \\ ${ }^{1}$ Hematology and Oncology Research Center, Tabriz University of Medical Sciences, Tabriz 5166614711, Iran \\ ${ }^{2}$ School of Medicine, Tabriz University of Medical Sciences, Tabriz 5166614711, Iran \\ ${ }^{3}$ Liver and Gastrointestinal Disease Research Center, Tabriz University of Medical Sciences, Tabriz 5166614711, Iran \\ ${ }^{4}$ Tabriz Health Services Management Research Center and Department of Statistics and Epidemiology, Faculty of Health and Nutrition, \\ Tabriz University of Medical Sciences, Tabriz 5166614711, Iran \\ ${ }^{5}$ Nutrition Research Center, Tabriz University of Medical Sciences, Tabriz 5166614711, Iran \\ ${ }^{6}$ Common Diseases Risk Factors Management Institute, Tabriz University of Medical Sciences, Tabriz 5166614711, Iran
}

Correspondence should be addressed to Zohreh Ghoreishi; zohreh_ghoreishy@yahoo.com

Received 30 August 2015; Accepted 24 December 2015

Academic Editor: Helieh $\mathrm{Oz}$

Copyright (C) 2016 Ali Esfahani et al. This is an open access article distributed under the Creative Commons Attribution License, which permits unrestricted use, distribution, and reproduction in any medium, provided the original work is properly cited.

\begin{abstract}
Background. There is a considerable dissimilarity in the survival duration of the patients with gastric cancer. We aimed to assess the systemic inflammatory response (SIR) and nutritional status of these patients before the commencement of chemotherapy to find the appropriate prognostic factors and define a new score for predicting metastasis. Methods. SIR was assessed using Glasgow Prognostic Score (GPS). Then a score was defined as prealbumin/CRP based prognostic score (PCPS) to be compared with GPS for predicting metastasis and nutritional status. Results. 71 patients with gastric cancer were recruited in the study. $87 \%$ of patients had malnutrition. There was a statistical difference between those with metastatic $(n=43)$ and those with nonmetastatic $(n=28)$ gastric cancer according to levels of prealbumin and CRP; however they were not different regarding patient generated subjective global assessment (PG-SGA) and GPS. The best cut-off value for prealbumin was determined at $0.20 \mathrm{mg} / \mathrm{dL}$ and PCPS could predict metastasis with $76.5 \%$ sensitivity, $63.6 \%$ specificity, and $71.4 \%$ accuracy. Metastatic and nonmetastatic gastric cancer patients were different in terms of PCPS $(P=0.005)$. Conclusion. PCPS has been suggested for predicting metastasis in patients with gastric cancer. Future studies with larger sample size have been warranted.
\end{abstract}

\section{Introduction}

The majority of patients with gastric cancer have a poor overall survival. Therefore, finding the appropriate prognostic factors will help to improve clinical approach on patients that will lead to accurate decision making and planning for supportive care. This will possibly increase the rate of survival [1].

Weight loss and performance status are usually used to predict survival and treatment outcomes in patients with inoperable gastric adenocarcinoma [2], but the degree to which they are associated with poor prognosis is not well defined and performance status does not provide an objective measurement $[3,4]$. Studies have shown that the presence of malnutrition and a systematic inflammatory response cause a short survival, reduced response rate, and higher risk for treatment-induced complications in patients with malignancy $[5,6]$.

Recently, the host nutritional and immune status have been evaluated by the Glasgow Prognostic Score (GPS), which is a combination of serum C-reactive protein (CRP) as an index for systemic inflammatory response and an important factor for the development and progression of neoplasms [7] along with serum albumin which has been proposed as a prognostic factor in a variety of cancers. GPS has prognostic importance independent of tumor stage in number of malignancies including gastrointestinal cancer $[4,8-10]$.

On the other hand, prealbumin is a remarkable prognostic factor for treatment outcomes and/or nutritional 
TABLE 1: General characteristics of the patients and disease.

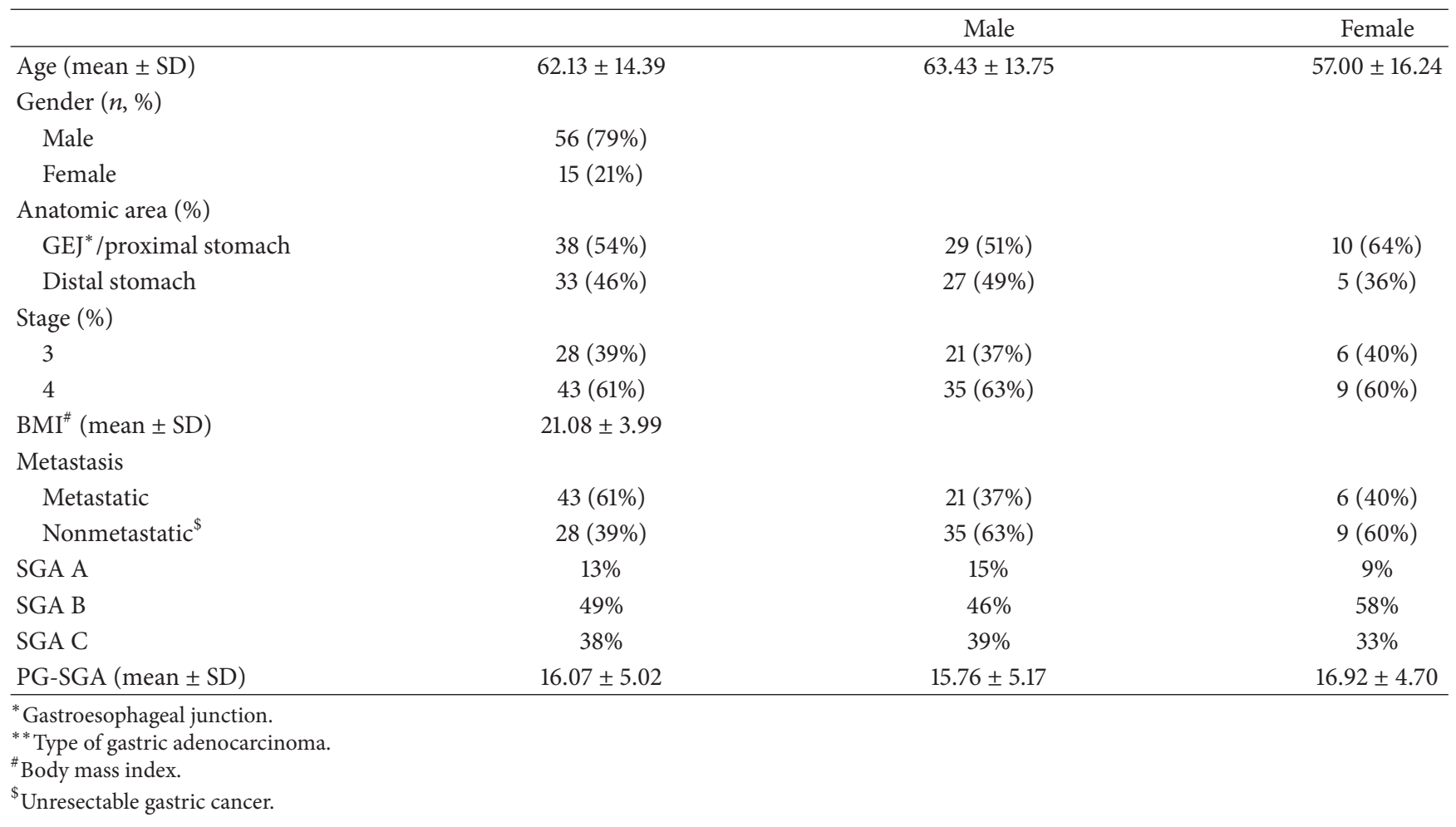

status of colon [11], esophagus [12], ovarian [13], and lung cancers $[14,15]$. Recently, we studied the nutritional status of patients with acute lymphoblastic leukemia (ALL) and acute myeloid leukemia (AML) during induction chemotherapy and its effects on chemotherapy-related complications in which prealbumin was found as the common biomarker for better treatment outcomes in both groups of patients with acute leukemia [16]. Here, systemic inflammatory response of the patients with inoperable gastric adenocarcinoma (IGA) was investigated by GPS, while their nutritional status was assessed using patient generated subjective global assessment (PG-SGA) as well as the serum levels of albumin, prealbumin, transferrin, CRP, and total lymphocyte count (TLC). Then a new prognostic score, prealbumin/CRP based prognostic score (PCPS), was introduced for predicting metastasis in this group of patients based on serum prealbumin and CRP and compared with PG-SGA and GPS.

\section{Materials and Methods}

2.1. Study Population. A convenient sample of 71 patients with inoperable gastric adenocarcinoma was recruited in this prospective study before the onset of chemotherapy between February 2013 and March 2014 (Table 1). The Human Ethics Committee of Tabriz University of Medical Sciences approved the study and written informed consent was obtained from all the patients before the commencement of the study. Patients with history of other malignancies, autoimmune disease, chronic renal or hepatic disease, diabetes, and thyroid disorders and those who were taking anti-inflammatory drugs were excluded from the study. The
Tumor-Node-Metastasis (TNM) classification of malignant tumors was used for staging the tumors. All patients received the same chemotherapy regimen as described below.

The chemotherapy regimen was as follows: docetaxel, $75 \mathrm{mg} / \mathrm{m}^{2}$ i.v. (one-hour infusion) on day 1; cisplatin, $75 \mathrm{mg} / \mathrm{m}^{2}$ i.v. (one-hour infusion) on day 1 ; and 5-fluorouracil (5-FU) i.v. (continuous infusion) on days $1-5$, to be repeated every 3 weeks for 6 cycles.

2.2. Biochemical Analyses. Venus blood samples were taken after an overnight fasting and the serum was separated and stored at $-70^{\circ} \mathrm{C}$ for future analysis. Hitachi 917 automated equipment was used for measuring albumin concentration and serum CRP, prealbumin, and transferrin were analyzed using the Minineph Human kits (Birmingham, UK).

2.3. Immunological Analyses. The systemic inflammatory response was measured using a combination of serum Creactive protein and albumin as follows: patients with $\mathrm{C}$ reactive protein $\leq 10 \mathrm{mg} / \mathrm{L}$ and albumin $\geq 3.5 \mathrm{mg} / \mathrm{dL}$ were allocated a score of 0 ; patients with one of these parameters abnormalities were allocated a score of 1 ; and those with both abnormalities, C-reactive protein $>10 \mathrm{mg} / \mathrm{L}$ and albumin $<3.5 \mathrm{~g} / \mathrm{dL}$, were allocated a score of two (Table 2). Then a new score was defined and called the prealbumin/CRP based prognostic score (PCPS) and it was compared with conventional GPS for evaluating the inflammatory status of patients and predicting metastasis. The PCPS was constructed using prealbumin and C-reactive protein with the same cut-off value for CRP and $0.20 \mathrm{mg} / \mathrm{dL}$ for prealbumin. 
TABLE 2: Classification of prealbumin/CRP based prognostic score (PCPS).

\begin{tabular}{lcc}
\hline Prealbumin $(\mathrm{mg} / \mathrm{dL})$ & CRP $(\mathrm{mg} / \mathrm{L})$ & PCPS \\
\hline $0.20<$ & $<10$ & 0 \\
$0.20<$ & $10 \leq$ & 1 \\
$<0.20$ & $<10$ & 1 \\
$<0.20$ & $10 \leq$ & 2 \\
\hline
\end{tabular}

Similar categorization was used for allocating scores of 0,1 , and 2 to the patients.

2.4. Nutritional Assessment. BMI was computed as weight $(\mathrm{kg}) /$ height $\left(\mathrm{m}^{2}\right)$. The scored PG-SGA was completed by all the patients with the help of a trained oncology nurse. PGSGA consists of the history of weight changes, food intakes, and the contributing factors, activities, physical examination, and the metabolic stress which affects the nutritional requirements. Based on scored PG-SGA, patients were scored at $0-1$ (with no need of nutritional intervention) and there was a progressive need for nutritional support, so that those who scored $\geq 9$ required immediate symptom management and/or nutritional support. PG-SGA also provided a categorical assessment as PG-SGA A (well-nourished), PG-SGA B (moderate malnutrition), and PG-SGA C (severe malnutrition) [5].

2.5. Statistical Analyses. Quantitative variables were presented as mean (standard deviation [SD]) or median (percentile 25-percentile 75) based on the normality of the distribution, and qualitative variables were reported as frequency (\%). The best cut-off point value for prealbumin was determined using receiver operating characteristic (ROC) analysis, considering the optimal sensitivity and specificity to calculate the Youden Index ((specificity + sensitivity) -1$)$. The likelihood ratios (LRs) and area under curve (AUC) were shown as a measure of metastasis prediction adequacy using prealbumin concentration. Then regression tree analysis was used to measure sensitivity, specificity, and accuracy of the new score, PCPS, for prediction of metastasis in IGA patients.

The association between categorized variables was examined using Chi-square test or Fisher exact test. Independentsamples Kruskal-Wallis test was used to determine the association between scored PG-SGA and categorized variables.

The significance level was considered 0.05 by doing a twotailed analysis. SPSS software (SPSS Inc., Chicago, IL) was used for performing statistical analyses.

\section{Results}

General characteristics of patients are shown in Table 1. Seventy-nine percent $(n=56)$ of the patients were male and $21 \%(n=15)$ were female with an average of $62.13 \pm 14.39$ and $21.08 \pm 3.99$ for age and BMI, respectively. Twenty-eight patients (39\%) had locally advanced unresectable gastric cancer (stage 3) (Figure 1) and 43 patients (61\%) had distance metastasis (stage 4) (Figure 2). According to categorized PG-SGA, 13\% of patients were well-nourished (PG-SGA A),

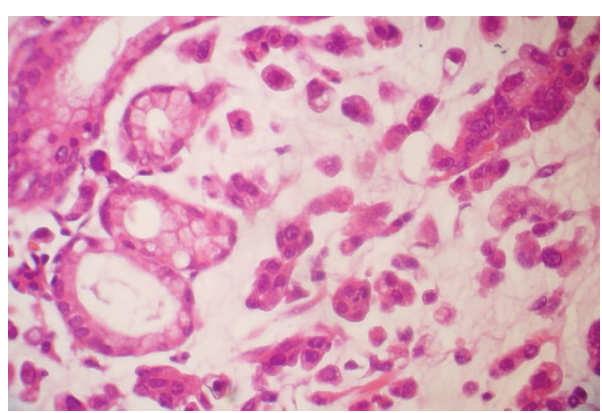

Figure 1: Microscopic scheme of metastatic diffuse type gastric cancer.

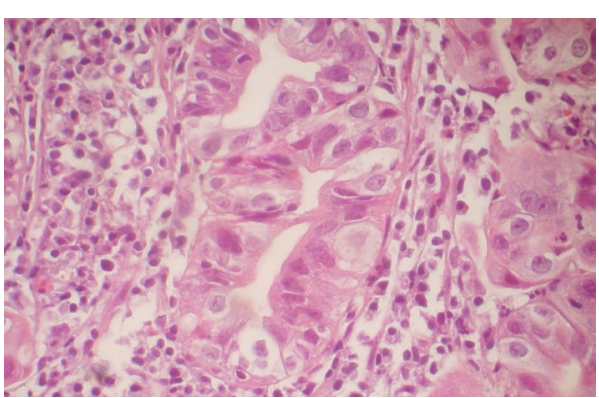

FIGURE 2: Microscopic scheme of nonmetastatic intestinal type gastric cancer.

while $49 \%$ of them were moderately (PG-SGA B) and $38 \%$ severely (PG-SGA C) malnourished. Considering PG-SGA $\mathrm{A}$ as an index for well-nourished category and PG-SGA B and PG-SGA C for some degrees of malnutrition, $87 \%$ of patients suffered from malnutrition before the beginning of chemotherapy. The mean score for PG-SGA was $16.07 \pm 5.02$, an indicative of need for immediate nutritional support.

Then metastatic and nonmetastatic patients were compared in terms of BMI, scored PG-SGA, visceral proteins, CRP, and TLC. There was a statistical difference between them according to prealbumin and CRP; $P=0.012$ and $P=0.004$, respectively (Table 3 ).

The best cut-off value for prealbumin was determined at $0.20 \mathrm{mg} / \mathrm{dL}$ for differentiating metastatic from nonmetastatic status using ROC analysis (Table 4). Using regression tree analysis, PCPS could predict metastasis with $76.5 \%$ sensitivity, $63.6 \%$ specificity, and $71.4 \%$ accuracy (considering scores 0 and 1 in one category and score 2 in the second category).

There was no statistical association between GPS, PCPS, and categorized PG-SGA, although $72 \%$ of the patients with severe malnutrition had a score of 2 for PCPS (Table 5). Moreover, the distribution of scored PG-SGA was the same across the categories of GPS and PCPS $(P=0.527$ and $P=0.334)$.

There was a statistical difference in PCPS score between metastatic and nonmetastatic gastric cancer patients $(P=$ 0.009), while they were not different in terms of GPS (Table 6). Moreover, there was no statistical association between anatomic location and site of gastric adenocarcinoma and PCPS $(P=0.701$ and $P=0.956$, resp. $)$. 
TABLE 3: Comparison between patients with metastatic and nonmetastatic inoperable gastric adenocarcinoma based on indicators of nutritional and inflammatory status.

\begin{tabular}{lcccc}
\hline & Metastatic & Nonmetastatic $^{\$}$ & Mean difference $(95 \% \mathrm{CI})$ & $P$ value \\
\hline BMI $^{*}$ & $21.94 \pm 3.94$ & $22.13 \pm 4.11$ & $0.18(-2.43$ to 2.81$)$ & 0.885 \\
Albumin $^{*}$ & $3.57 \pm 0.74$ & $3.94 \pm 0.68$ & $0.36(-0.30$ to 0.76$)$ & $0.06(0.01$ to 0.09$)$ \\
Prealbumin $^{*}$ & $0.14 \pm 0.06$ & $0.20 \pm 0.10$ & $60.59(-5.75$ to 126.94$)$ & 0.012 \\
Transferrin $^{*}$ & $218.48 \pm 119.68$ & $279.07 \pm 135.75$ & & 0.073 \\
CRP $^{* *}$ & $37.60(15.59-85.16)$ & $15.61(5.52-30.01)$ & $0.09(-0.19$ to 0.37$)$ & 0.004 \\
TLC $^{*}$ & $1.18 \pm 0.49$ & $1.27 \pm 0.54$ & 0.564 & 0.564 \\
PG-SGA $^{* *}$ & $17(13-20.50)$ & $17(11-19)$ &
\end{tabular}

${ }^{*}$ Mean $\pm \mathrm{SD}, P$ value based on independent-samples $t$-test.

** Median (percentiles 25-75), $P$ value based on Mann-Whitney test (only $P$ value was reported).

${ }^{\$}$ Unresectable gastric cancer.

TABLE 4: Receiver operating characteristic (ROC) analysis and optimum cut-off point of prealbumin for predicting metastasis in patients with inoperable gastric adenocarcinoma.

\begin{tabular}{ccccccccc}
\hline & PA & AUC & SEN & SPE & PPV & NPV & LR $^{+}$ & LR $^{-}$ \\
\hline \multirow{2}{*}{ mets } & 0.20 & 0.68 & $77.1 \%$ & $52.2 \%$ & $71.1 \%$ & $60.0 \%$ & 1.61 & 0.44 \\
& & $(0.54-0.82)^{*}$ & $(61.0-87.9)$ & $(33.0-70.8)$ & $(55.2-83.0)$ & $(38.7-78.1)$ & $(1.01-2.56)$ & $(0.21-0.90)$ \\
\hline
\end{tabular}

Mets: metastasis; ${ }^{*}$ 95\% confidence interval (CI); PA: prealbumin (mg/dL); AUC: area under the curve; SEN: sensitivity; SPE: specificity; LR $^{+}$: positive likelihood ratio; $\mathrm{LR}^{-}$: negative likelihood ratio; NPV: negative predictive value; PPV: positive predictive value.

TABLE 5: The association between GPS, PCPS, and categorized PGSGA.

\begin{tabular}{ccccc}
\hline & $\begin{array}{c}\text { PG-SGA } \\
\text { A (\%) }\end{array}$ & $\begin{array}{c}\text { PG-SGA } \\
\text { B (\%) }\end{array}$ & $\begin{array}{c}\text { PG-SGA } \\
\text { C (\%) }\end{array}$ & $P$ value** \\
\hline GPS $^{\#}$ & & & & \\
0 & 40 & 19 & 33 & \\
1 & 20 & 52 & 25 & \\
2 & 40 & 29 & 42 & 0.527 \\
PCPS $^{\$}$ & & & & \\
0 & 40 & 14 & 14 & \\
1 & 40 & 19 & 14 & \\
2 & 20 & 67 & 72 & 0.334 \\
\hline
\end{tabular}

${ }^{*}$ Patient generated subjective global assessment.

${ }^{* *} P$ value was calculated based on independent-samples Kruskal-Wallis test.

\# Glasgow Prognostic Score.

${ }^{\$}$ Prealbumin/CRP based prognostic score.

\section{Discussion}

Malnutrition and systemic inflammatory response are common in patients with cancer and they both have significant impact on patients' quality of life, treatment outcomes, prognosis, and survival $[4,5,15,17]$. In this study, the nutritional and inflammatory status of patients with inoperable gastric adenocarcinoma were assessed using known scores of PGSGA, a valid tool for nutritional assessment of patients with cancer and GPS, a score for measuring systemic inflammation, and an independent prognostic score in different kinds of malignancies including gastrointestinal cancer $[4,8-10]$. Eighty-seven percent of patients had some degrees of malnutrition and $65 \%$ of them had GPS scores of 1 or 2 , but there was
TABLE 6: The differences between metastatic and nonmetastatic patients with inoperable gastric adenocarcinoma in terms of PGSGA, GPS, and PCPS.

\begin{tabular}{cccc}
\hline & Metastatic & Nonmetastatic & $P$ value \\
\hline PG-SGA $^{* *}(\%)$ & & & \\
A & 12 & 19 & \\
B & 48 & 44 & 0.820 \\
C & 40 & 37 & \\
GPS $^{\#}(\%)$ & & & \\
0 & 16 & 36 & 0.153 \\
1 & 40 & 41 & \\
2 & 44 & 23 & \\
PCPS $^{\$}(\%)$ & & & \\
0 & 9 & 27 & $\mathbf{0 . 0 0 9}$ \\
1 & 15 & 37 & \\
2 & 76 & 36 & \\
\hline
\end{tabular}

${ }^{*}$ Patient generated subjective global assessment.

${ }^{* *} P$ value was calculated based on exact Chi-square test.

\# Glasgow Prognostic Score.

${ }^{\$}$ Prealbumin/CRP based prognostic score.

no significant difference between patients with metastatic and nonmetastatic gastric cancer in terms of PG-SGA or GPS.

Comparing patients with metastatic and nonmetastatic gastric cancer, it was found that they were different statistically according to prealbumin and CRP and not albumin. The results of this study confirm the findings of previous studies that showed that baseline levels of prealbumin had a significant correlation to overall survival in patients with advanced colorectal cancer [11] and esophageal cancer [12]. In a study 
on patients with non-small cell lung cancer [15] and epithelial ovarian carcinoma [18] prechemotherapy concentrations of prealbumin were associated with response to treatment and outcomes. Moreover, Ho et al. found that low level of prealbumin was an independent prognostic factor for overall survival in cancer patients and its assessment has been suggested to be considered as a part of palliative care setting [18].

Notably, Inoue et al. investigated the association between the serum levels of rapid turnover proteins (RTPs) and the prognosis in patients with advanced cancer receiving total parenteral nutrition. They found that there was a significant association between RTPs' concentration and survival in cancer patients and among RTPs and prealbumin had the most correct prognosis rate with $91.9 \%$ compared to transferrin and retinol binding protein [19]. The short prealbumin halflife of $\approx 2$ days in the blood circulation makes it a more sensitive biomarker for the assessment of nutritional state compared to albumin with much longer half-life [20, 21]. It should be mentioned that we studied the nutritional status of patients with ALL and AML during induction chemotherapy and its impact on chemotherapy-related complications in which prealbumin was the common biomarker for better treatment outcomes in both groups of patients with acute leukemia [16].

On the other hand, CRP is an indicator of systemic inflammatory response and studies have shown the independent prognostic value of elevated serum levels of CRP in solid tumors including gastroesophageal cancer [6]. Given the evidences, this study designed a new prognostic score based on prechemotherapy concentrations of prealbumin and CRP, after determining the best cut-off point value of prealbumin by ROC analysis at $0.20 \mathrm{mg} / \mathrm{dL}$ for differentiating metastatic from nonmetastatic status named PCPS. This score could predict metastasis with $76.5 \%$ sensitivity, $63.6 \%$ specificity, and $71.4 \%$ accuracy. Noteworthy, the patients with metastatic and nonmetastatic gastric cancer were significantly different according to PCPS unlike the conventional score of GPS. However, there was no significant association between GPS, PCPS, and categorized PG-SGA which may be due to the relatively small sample size and the fact that $87 \%$ of the patients (metastatic and nonmetastatic) already had malnutrition (before the onset of chemotherapy). So PCPS could not be considered as a tool for assessment of nutritional status in patients with inoperable gastric adenocarcinoma.

The small sample size was one of the possible limitations, as it was a single center study and a large number of eligible patients declined inclusion as a result of their critical conditions. Moreover, there was no official registration system, so the patients could not be followed to determine the survival rate. However, this study presented a composite score of prealbumin and CRP suggesting that it may be a strong prognostic score in patients with inoperable gastric adenocarcinoma. Although it is early to propose the replacement of GPS with PCPS, assessment of the host inflammatory response and nutritional status with PCPS and evaluation of its association with response to treatment, prognosis, complications, and survival in patients with different kinds of cancer is warranted.

\section{Abbreviations}

$\begin{array}{ll}\text { GPS: } & \text { Glasgow Prognostic Score } \\ \text { CRP: } & \text { C-reactive protein } \\ \text { ALL: } & \text { Acute lymphoblastic leukemia } \\ \text { AML: } & \text { Acute myeloid leukemia } \\ \text { PG-SGA: } & \text { Patient generated subjective global assessment } \\ \text { TLC: } & \text { Total lymphocyte count } \\ \text { PCPS: } & \text { Prealbumin/CRP based prognostic score } \\ \text { RTPs: } & \text { Rapid turnover proteins. }\end{array}$

\section{Conflict of Interests}

The authors declare that there is no conflict of interests.

\section{References}

[1] A. B. C. Crumley, D. C. McMillan, M. McKernan, A. C. McDonald, and R. C. Stuart, "Evaluation of an inflammationbased prognostic score in patients with inoperable gastrooesophageal cancer," British Journal of Cancer, vol. 94, no. 5, pp. 637-641, 2006.

[2] I. Chau, A. R. Norman, D. Cunningham, J. S. Waters, J. Oates, and P. J. Ross, "Multivariate prognostic factor analysis in locally advanced and metastatic esophago-gastric cancerpooled analysis from three multicenter, randomized, controlled trials using individual patient data," Journal of Clinical Oncology, vol. 22, no. 12, pp. 2395-2403, 2004.

[3] M. Ando, Y. Ando, Y. Hasegawa et al., "Prognostic value of performance status assessed by patients themselves, nurses, and oncologists in advanced non-small cell lung cancer," British Journal of Cancer, vol. 85, no. 11, pp. 1634-1639, 2001.

[4] D. C. McMillan, "Systemic inflammation, nutritional status and survival in patients with cancer," Current Opinion in Clinical Nutrition \& Metabolic Care, vol. 12, no. 3, pp. 223-226, 2009.

[5] J. Bauer, S. Capra, and M. Ferguson, "Use of the scored Patient-Generated Subjective Global Assessment (PG-SGA) as a nutrition assessment tool in patients with cancer," European Journal of Clinical Nutrition, vol. 56, no. 8, pp. 779-785, 2002.

[6] A. B. C. Crumley, D. C. McMillan, M. McKernan, J. J. Going, C. J. Shearer, and R. C. Stuart, "An elevated C-reactive protein concentration, prior to surgery, predicts poor cancerspecific survival in patients undergoing resection for gastrooesophageal cancer," British Journal of Cancer, vol. 94, no. 11, pp. 1568-1571, 2006.

[7] C. S. D. Roxburgh and D. C. McMillan, "Role of systemic inflammatory response in predicting survival in patients with primary operable cancer," Future Oncology, vol. 6, no. 1, pp. 149163, 2010.

[8] D. C. McMillan, "An inflammation-based prognostic score and its role in the nutrition-based management of patients with cancer," Proceedings of the Nutrition Society, vol. 67, no. 3, pp. 257-262, 2008

[9] M. J. Proctor, D. S. Morrison, D. Talwar et al., "An inflammationbased prognostic score (mGPS) predicts cancer survival independent of tumour site: a Glasgow Inflammation Outcome Study," British Journal of Cancer, vol. 104, no. 4, pp. 726-734, 2011.

[10] T. Ikeya, M. Shibutani, K. Maeda et al., "Maintenance of the nutritional prognostic index predicts survival in patients with unresectable metastatic colorectal cancer," Journal of Cancer Research and Clinical Oncology, vol. 141, no. 2, pp. 307-313, 2015. 
[11] P. Byström, Å. Berglund, P. Nygren et al., "Evaluation of predictive markers for patients with advanced colorectal cancer," Acta Oncologica, vol. 51, no. 7, pp. 849-859, 2012.

[12] P. Kelly, F. Paulin, D. Lamont et al., "Pre-treatment plasma proteomic markers associated with survival in oesophageal cancer," British Journal of Cancer, vol. 106, no. 5, pp. 955-961, 2012.

[13] C.-G. Mahlck and K. Granvist, "Plasma prealbumin in women with epithelial ovarian carcinoma," Gynecologic and Obstetric Investigation, vol. 37, no. 2, pp. 135-140, 1994.

[14] M. Alifano, A. Mansuet-Lupo, F. Lococo et al., "Systemic inflammation, nutritional status and tumor immune microenvironment determine outcome of resected non-small cell lung cancer," PLoS ONE, vol. 9, no. 9, Article ID e106914, 2014.

[15] H. Kawai and H. Ota, "Low perioperative serum prealbumin predicts early recurrence after curative pulmonary resection for non-small-cell lung cancer," World Journal of Surgery, vol. 36, no. 12, pp. 2853-2857, 2012.

[16] A. Esfahani, Z. Ghoreishi, M. Abedi Miran et al., "Nutritional assessment of patients with acute leukemia during induction chemotherapy: association with hospital outcomes," Leukemia \& Lymphoma, vol. 55, no. 8, pp. 1743-1750, 2014.

[17] J. A. Read, S. T. B. Choy, P. Beale, and S. J. Clarke, "An evaluation of the prevalence of malnutrition in cancer patients attending the outpatient oncology clinic," Asia-Pacific Journal of Clinical Oncology, vol. 2, no. 2, pp. 80-86, 2006.

[18] S.-Y. Ho, H.-R. Guo, H. H. W. Chen, and C.-J. Peng, "Nutritional predictors of survival in terminally ill cancer patients," Journal of the Formosan Medical Association, vol. 102, no. 8, pp. 544-550, 2003.

[19] Y. Inoue, R. Nezu, H. Matsuda, Y. Takagi, and A. Okada, "Rapid turnover proteins as a prognostic indicator in cancer patients," Surgery Today, vol. 25, no. 6, pp. 498-506, 1995.

[20] F. K. Beck and T. C. Rosenthal, "Prealbumin: a marker for nutritional evaluation," American Family Physician, vol. 65, no. 8, pp. 1575-1578, 2002.

[21] G. Devoto, F. Gallo, C. Marchello et al., "Prealbumin serum concentrations as a useful tool in the assessment of malnutrition in hospitalized patients," Clinical Chemistry, vol. 52, no. 12, pp. 2281-2285, 2006. 


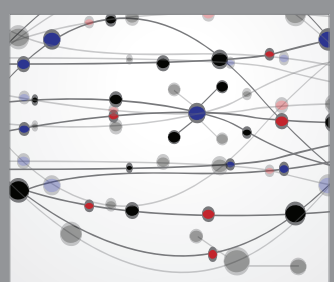

The Scientific World Journal
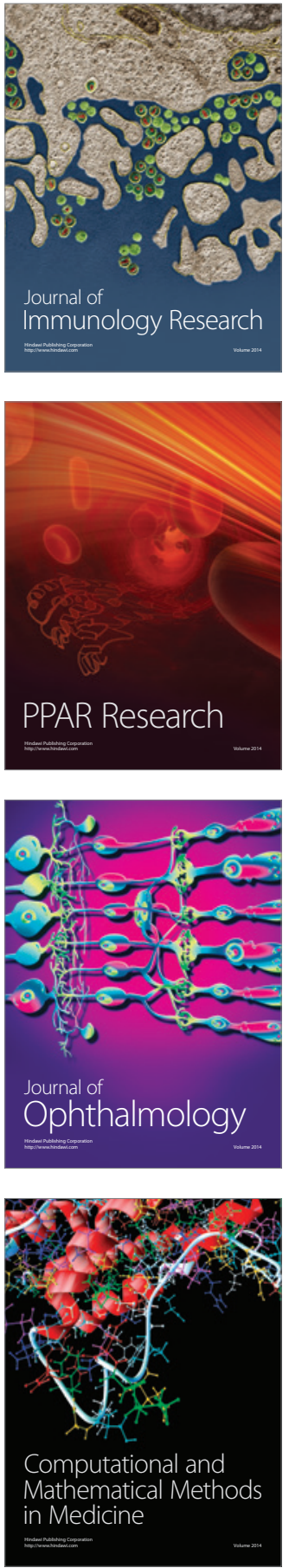

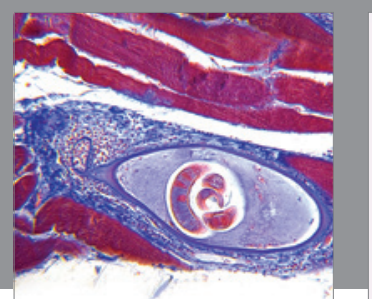

Gastroenterology Research and Practice

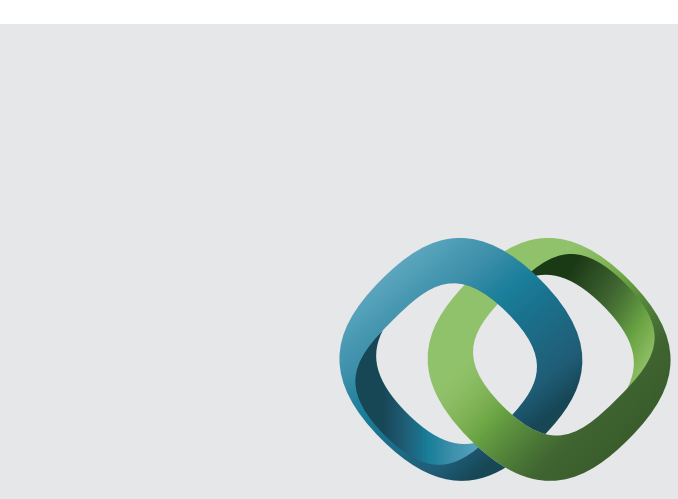

\section{Hindawi}

Submit your manuscripts at

http://www.hindawi.com
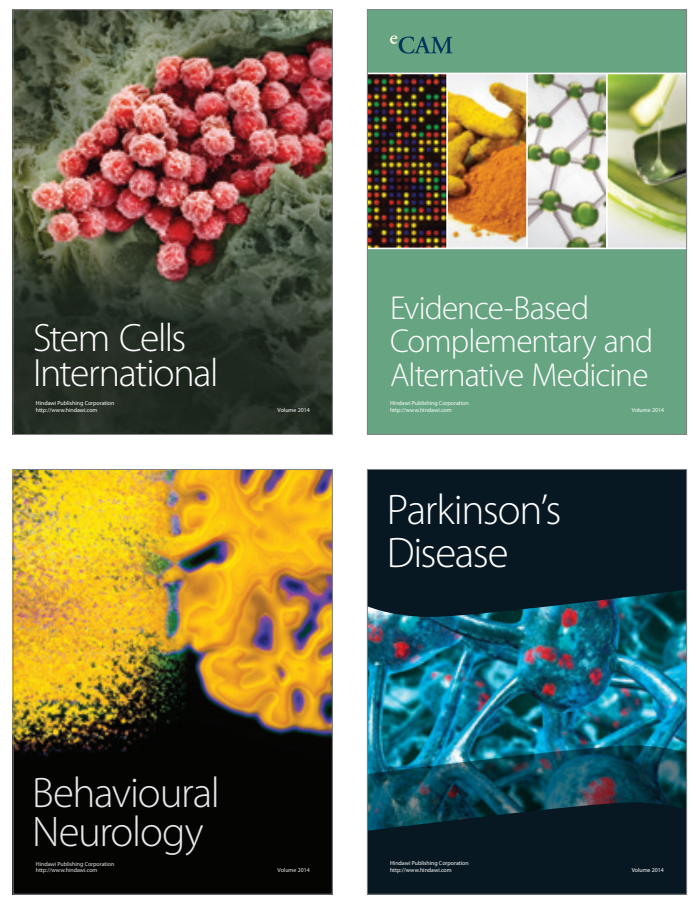
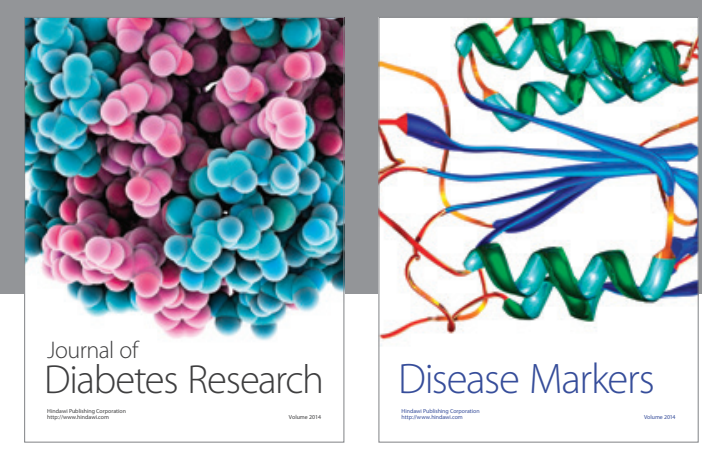

Disease Markers
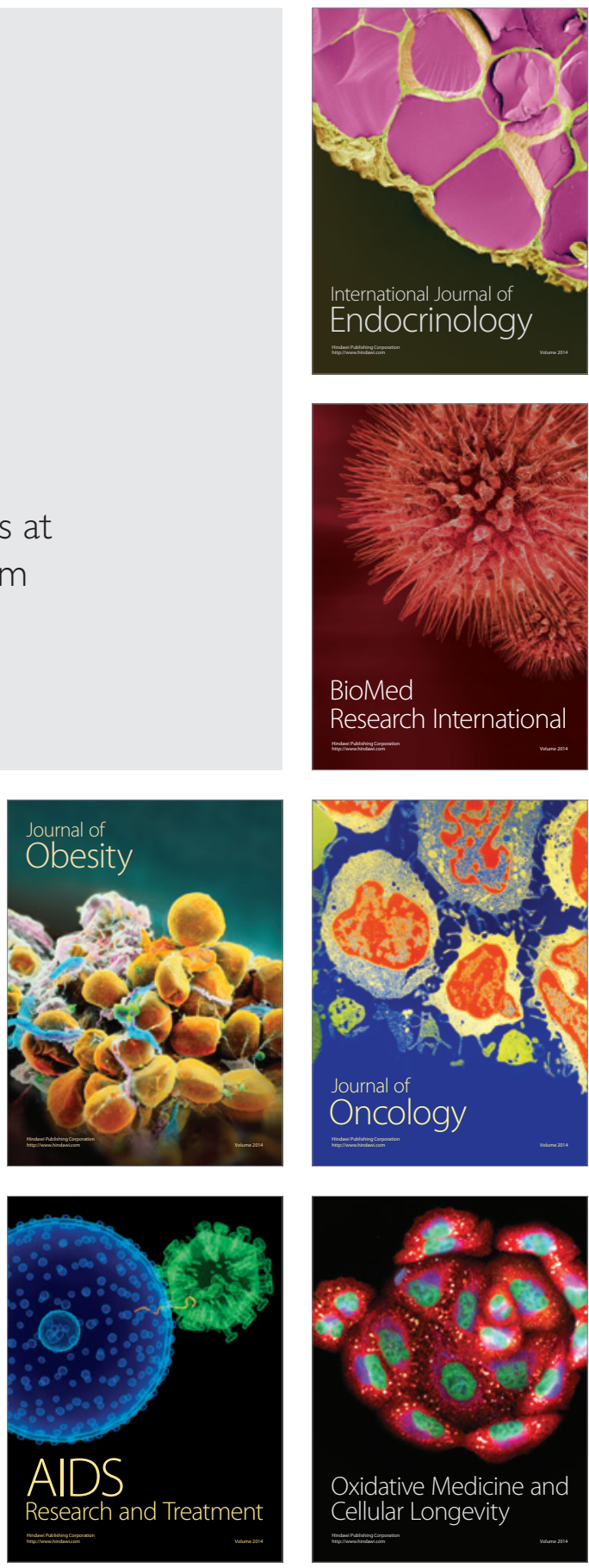